\title{
Lúpus Eritematoso Cutâneo Subagudo Induzido por Amlodipina
}

\section{Amlodipine-Induced Subacute Cutaneous Lupus Erythematosus}

Carla Longras ${ }^{1 *}$, Pedro Costa Dias², Fontes Alves ${ }^{3}$, Joana Santos ${ }^{4}$, Joana Rocha ${ }^{5}$

*Autor Correspondente/Corresponding Author:

Carla Longras [carlalongras@hotmail.com]

\section{RESUMO}

O lúpus eritematoso cutâneo subagudo é uma variante de lúpus eritematoso cutâneo que se manifesta por lesões anulares ou psoriasiformes. Pode ser induzido por fármacos e tem, no geral, um bom prognóstico. Dados publicados sugerem que os bloqueadores dos canais de cálcio estão entre os vários medicamentos implicados no seu aparecimento.

Homem de 76 anos com antecedentes de doença coronária, urticária crónica e depressão, medicado com amlodipina após cateterismo cardíaco. Três meses depois desenvolveu lesões maculopapulares e placas anulares rosadas, ligeiramente descamativas e pruriginosas, que atingiam predominantemente o tronco e, em menor extensão, os membros. Negava outros sintomas. Foi realizado estudo analítico, biópsia cutânea e exame micológico. A histologia cutânea revelou aspetos compatíveis com lúpus eritematoso subagudo. Os restantes exames foram normais. Observou-se melhoria do quadro após corticoterapia oral e suspensão de amlodipina.

Com este caso pretende-se realçar a importância de estar alerta para toxidermias pouco frequentes que podem ser induzidas por fármacos de utilização corrente na prática clínica.

PALAVRAS-CHAVE: Amlodipina; Lúpus Eritematoso Cutâneo

1. Unidade de Saúde Familiar Lagoa, Unidade Local de Saúde de Matosinhos, Matosinhos, Portugal. 2. Unidade de Saúde Familiar Caravela, Unidade Local de Saúde de Matosinhos, Matosinhos, Portugal. 3. Unidade de Saúde Familiar Infesta, Unidade Local de Saúde de Matosinhos, Matosinhos, Portugal. 4. Serviço de Anatomia Patológica do Hospital Pedro Hispano, Unidade Local de Saúde de Matosinhos, Matosinhos, Portugal. 5. Serviço de Dermato-Venereologia no Hospital Pedro Hispano, Unidade Local de Saúde de Matosinhos, Matosinhos, Portugal. 


\section{ABSTRACT}

Subacute cutaneous lupus erythematosus is a subtype of cutaneous lupus erythematosus characterized by annular or psoriasiform lesions. In general, it has a good prognosis. Some drugs may trig this skin disease, as such as the calcium channel blockers.

A 76-year-old man with personal history of coronary heart disease, chronic urticaria and depression received amlodipine after cardiac catheterization. Three months later, he presented with a pink pruritic slightly scaly maculopapular skin eruption and annular plaques affecting mainly the trunk and the limbs to a lesser extent. No other symptom was reported. Extensive blood tests, skin biopsy and mycology exam were performed. The histological examination of the skin tissue was compatible with subacute lupus erythematosus. The remaining laboratory study was normal. Remarkable improvement was seen after systemic steroid therapy and withdrawal of amlodipine.

This case report emphasizes that is important to be on the lookout for uncommon skin reaction triggered by drugs widely used in clinical practice.

KEYWORDS: Amlodipine; Lupus Erythematosus, Cutaneous

\section{INTRODUÇÃO}

O lúpus eritematoso cutâneo subagudo (LECS) é uma variante de lúpus eritematoso cutâneo, que se manifesta clinicamente por lesões anulares ou psoriasiformes localizadas geralmente em zonas fotoexpostas. Pode estar associado a outras doenças autoimunes ou ser induzido e/ou agravado por alguns fármacos. Nos casos de toxidermia, sugere-se que a etiopatogénese esteja associada a um estado de fotossensibilidade em consequência da exposição ao medicamento num indivíduo imunogeneticamente predisposto. ${ }^{1-4,5}$

Dados publicados sugerem que os principais fármacos envolvidos incluem a hidroclorotiazida, a terbinafina, os antagonistas do fator de necrose tumoral (TNF), os antiepiléticos, os inibidores da bomba de protões, os anti-inflamatórios não esteroides, os bloqueadores dos canais de cálcio e os inibidores da enzima conversora de angiotensina. ${ }^{1-5}$

O diagnóstico de LECS induzido por fármacos pode ser desafiante, uma vez que os sintomas caracteristicamente aparecem meses após a introdução do medicamento.1-5 No geral, as lesões neste subtipo de LECS localizam-se na parte superior do corpo, incluindo a face, pescoço e tronco. Comparativamente ao LECS idiopático, a apresentação clínica tende a ser mais generalizada e, raramente, existe sintomatologia sistémica associada. ${ }^{1-5}$

No geral, o tratamento inclui corticoterapia sistémica e/ ou tópica e, obrigatoriamente, a suspensão do fármaco. Uma vez instituídas tais medidas terapêuticas os sintomas normalmente resolvem em semanas. ${ }^{1-5}$

Descreve-se um caso de LECS com uma apresentação clínica desafiante e desencadeado por amlodipina, um fármaco de utilização corrente na prática clínica.

\section{CASO CLÍNICO}

Homem de 76 anos, reformado. Família díade nuclear, ciclo de Duvall (fase VII), classe média de Graffar. Antecedentes pessoais de doença coronária, urticária crónica e depressão, medicado cronicamente com atorvastatina e lisinopril. Iniciou tratamento com amlodipina, pantoprazol, mononitrato de isossorbida e ticagrelor após cateterismo cardíaco em agosto de 2019.

No dia 25/10/2019 recorreu à consulta aberta da sua unidade de saúde familiar por dermatose eritematosa e pruriginosa envolvendo o tronco e os braços, com início

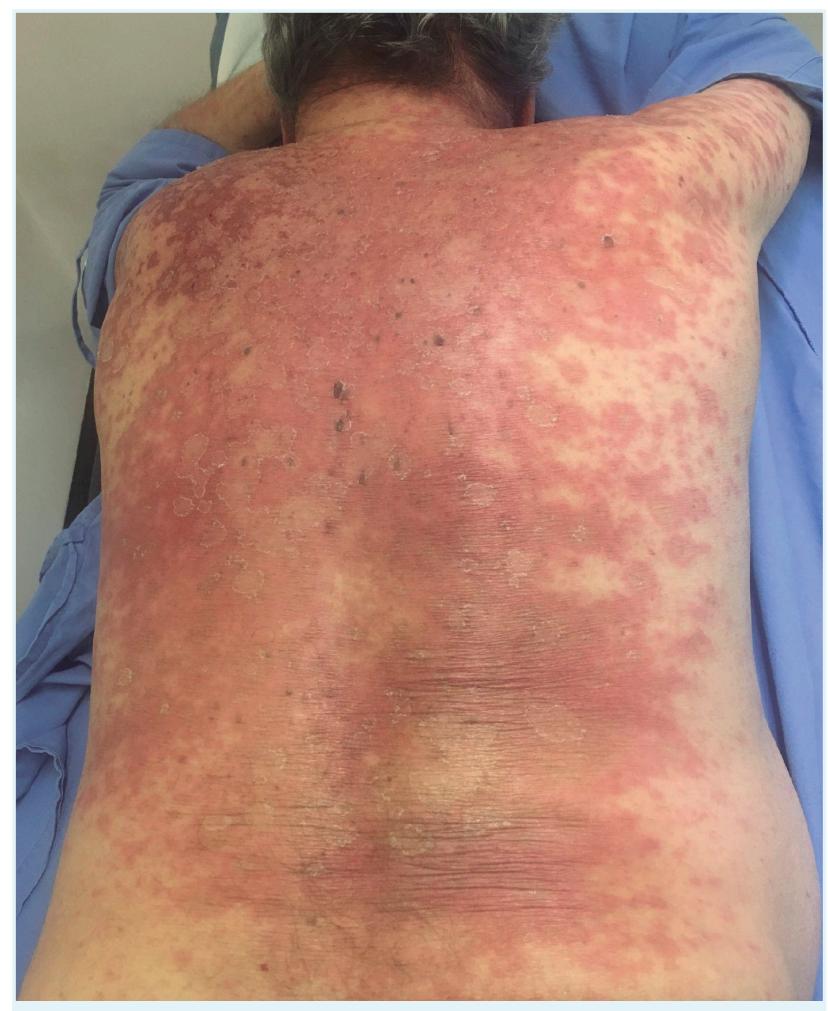

FIGURA 1. Placas anulares rosadas e ligeiramente descamativas. 


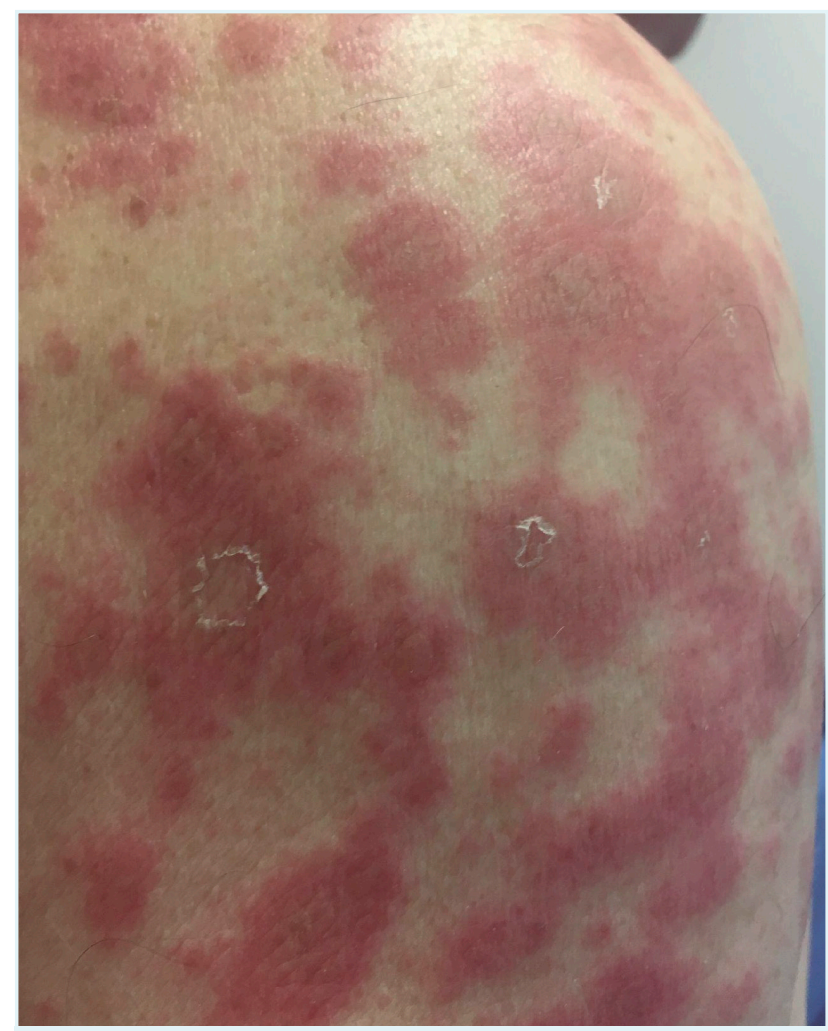

FIGURA 2. Placas anulares rosadas e ligeiramente descamativas.

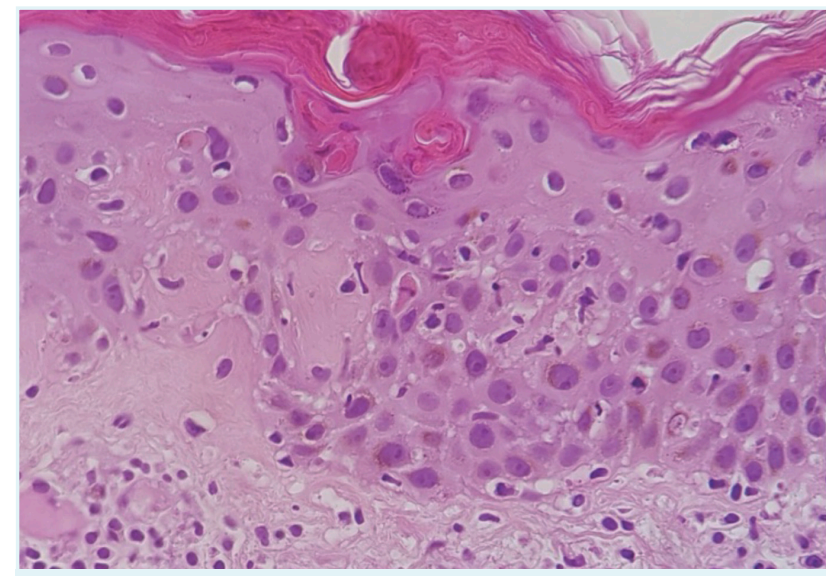

FIGURA 3. Exame histológico com paraqueratose, epidermotropismo, degenerescência hidrópica basal e infiltrado linfocitário perivascular sem eosinófilos.

no próprio dia. Ao exame objetivo o eritema desaparecia à digitopressão. As lesões individuais tinham duração superior a 24 horas. Foi medicado com bilastina $20 \mathrm{mg}$ id, durante 7 dias.

No dia 31/10/2019 foi observado em consulta de Cardiologia e por manutenção da dermatose foi pedida avaliação por Dermatologia. Iniciou-se tratamento com prednisolona $40 \mathrm{mg}$ id durante 3 dias, seguindo-se mais 3 dias com 30 mg id, tendo sido agendada reavaliação para 19/11/2019. Apesar do tratamento instituído, nessa consulta constatou-se persistência do quadro e presença de lesões anulares, rosadas, ligeiramente descamativas e pruriginosas, que atingiam o tronco e, em menor extensão, os membros (Fig.s 1 e 2). Negava

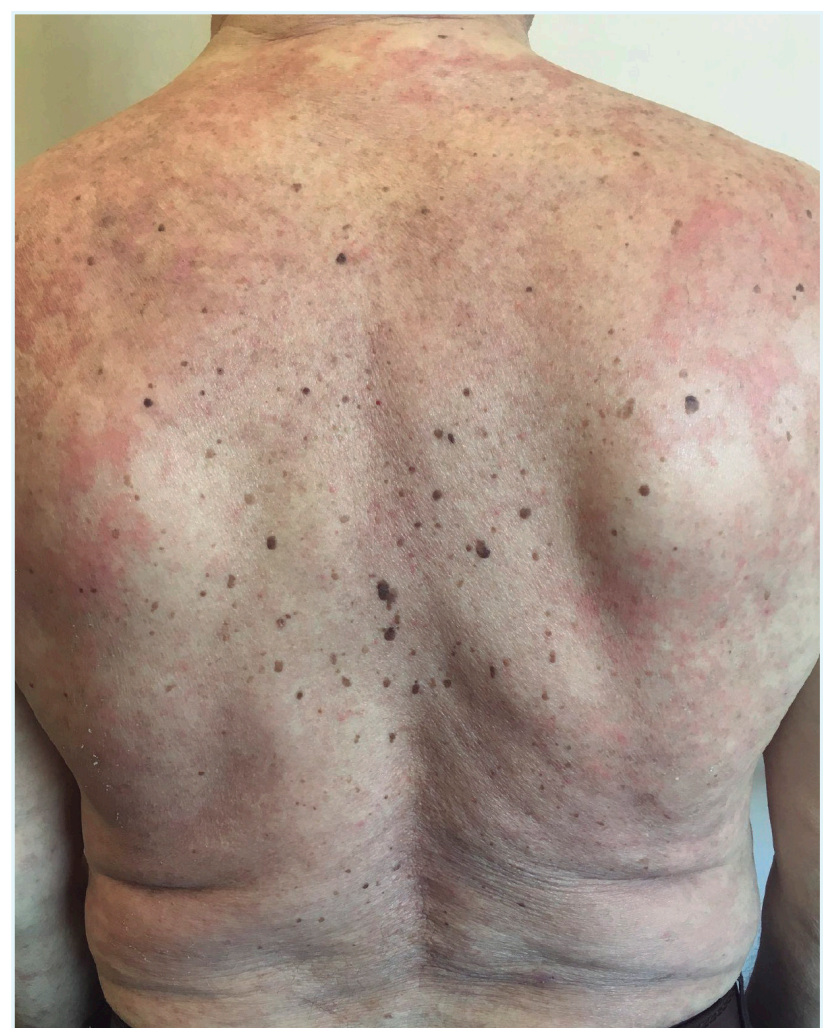

FIGURA 4. Lesões cutâneas em resolução.

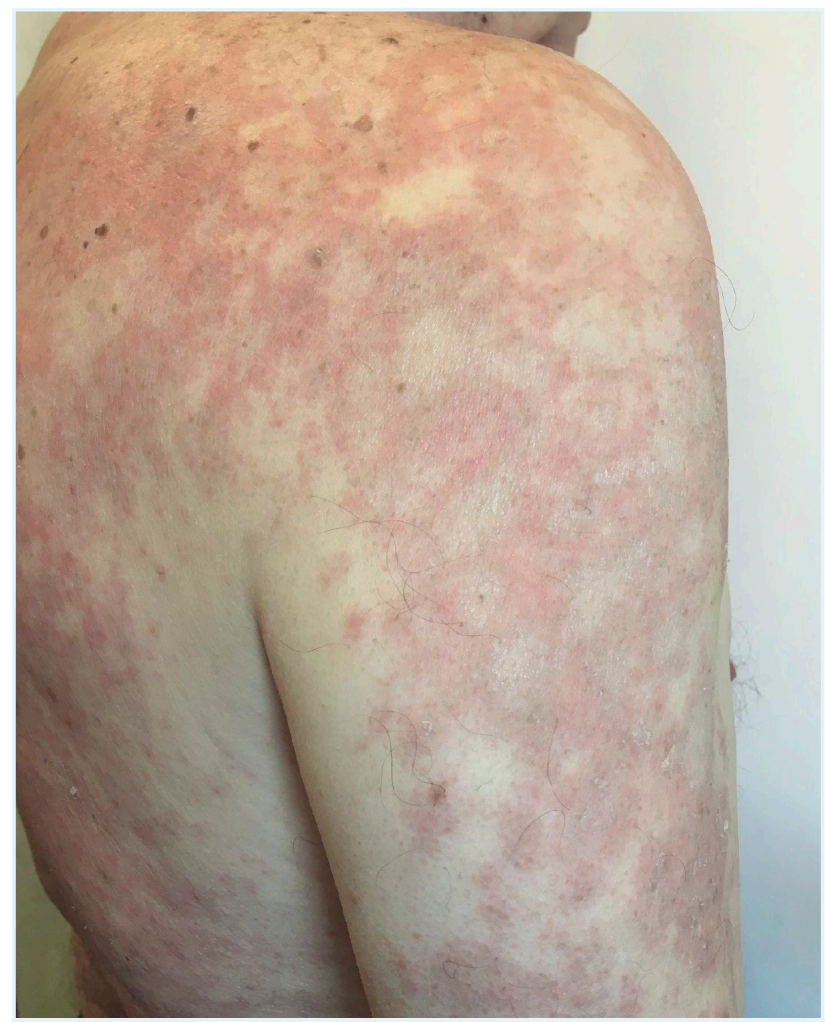

FIGURA 5. Lesões cutâneas em resolução.

outros sintomas. A face, as palmas, as plantas e as mucosas estavam poupadas. Foi realizado estudo analítico, colheita de escamas para exame micológico e biópsia cutânea para avaliação histológica e imunofluorescência direta. Nessa fase, as hipóteses de diagnóstico mais prováveis eram toxidermia, LECS e pênfigo foliáceo. 
O estudo analítico (incluindo os autoanticorpos) e o exame micológico foram negativos. O exame histológico de pele revelou epiderme com paraqueratose, queratinócitos apoptóticos basais dispersos, epidermotropismo e degenerescência hidrópica basal; na derme observou-se infiltrado linfocitário perivascular sem eosinófilos e incontinência pigmentar (Fig. 3). A imunofluorescência direta revelou depósito granular basal de C3. Estes achados eram compatíveis com LECS.

Neste contexto, estabeleceu-se o diagnóstico de LECS induzido por medicamento. Como referido anteriormente, o utente tinha começado amlodipina, pantoprazol, mononitrato de isossorbida e ticagrelor algumas semanas antes do início do quadro cutâneo. Dos quatro, o bloqueador do canal de cálcio e, menos provavelmente, o inibidor da bomba de protões, poderiam estar implicados no seu aparecimento. Desta forma, procedeu-se à suspensão da amlodipina e reintrodução de prednisolona oral $40 \mathrm{mg}$ id.

No dia 4/12/2020 foi observado novamente por Dermatologia e apresentava lesões em resolução (Fig.s 4 e 5). Foi iniciado um esquema de desmame da corticoterapia e agendada reavaliação dois meses depois.

\section{DISCUSSÃO}

Relatamos um caso de LECS induzido por amlodipina, uma dermatose pouco relatada na literatura do ponto de vista toxicológico. Não existem critérios definidos para o seu diagnóstico, sendo relevante a associação temporal e a resolução do quadro com a interrupção do medicamento suspeito. No caso relatado, o tipo de lesões que o doente apresentava e a sua distribuição, bem como o tempo entre a introdução do fármaco e o aparecimento das mesmas correspondiam ao descrito na literatura.

Os fármacos implicados no aparecimento do LECS são, no geral, de utilização comum na prática clínica diária, nomeadamente os diuréticos tiazídicos, os bloqueadores de canais de cálcio, os inibidores da enzima conversora da angiotensina e os inibidores da bomba de protões. ${ }^{2,5}$ Entre os segundos, o diltiazem é o mais frequente. ${ }^{5}$ Quando o doente tem prescrito mais do que um fármaco suspeito, a decisão sobre qual suspender primeiro pode ser desafiante. ${ }^{1}$ Neste caso clínico, optou-se pela amlodipina por haver registo anterior de toma do inibidor da bomba de protões sem reações adversas.

O principal diagnóstico diferencial do LECS induzido por fármacos é o LECS idiopático. No entanto, as apresentações clínicas, histopatológicas e imunopatológicas são semelhantes entre si. . $^{24-5}$ Desta forma, a resolução da dermatose com a cessação do fármaco suspeito é a melhor evidência diagnóstica. Alguns relatos demonstraram que a regressão das lesões geralmente ocorre em menos de três meses. ${ }^{2,4,5}$ Os restantes diagnósticos diferenciais incluem a psoríase, a dermatofitíase, o eritema anular centrífugo, a erupção medicamentosa liquenoide, o pênfigo foliáceo e o granuloma anular. 6

Realça-se a importância de estar alerta para a possibilidade de uma reação cutânea medicamentosa perante uma dermatose eritematosa, generalizada e pruriginosa, com início após introdução de novos fármacos. A história clínica, em particular a descrição detalhada da medicação habitual (nome, posologia, data do início e fim), é fundamental para o diagnóstico.

\section{RESPONSABILIDADES ÉTICAS}

CONFLITOS DE INTERESSE: Os autores declaram a inexistência de conflitos de interesse na realização do presente trabalho.

FONTES DE FINANCIAMENTO: Não existiram fontes externas de financiamento para a realização deste artigo.

CONFIDENCIALIDADE DOS DADOS: Os autores declaram ter seguido os protocolos da sua instituição acerca da publicação dos dados de doentes.

CONSENTIMENTO: Consentimento do doente para publicação obtido.

PROVENIÊNCIA E REVISÃO POR PARES: Não comissionado; revisão externa por pares.

\section{ETHICAL DISCLOSURES}

CONFLICTS OF INTEREST: The authors have no conflicts of interest to declare.

FINANCING SUPPORT: This work has not received any contribution, grant or scholarship.

CONFIDENTIALITY OF DATA: The authors declare that they have followed the protocols of their work center on the publication of data from patients.

PATIENT CONSENT: Consent for publication was obtained.

PROVENANCE AND PEER REVIEW: Not commissioned; externally peer reviewed.

\section{REFERÊNCIAS}

1. Sontheimer RD, Henderson CL, Grau RH. Drug-induced subacute cutaneous lupus erythematosus: a paradigm for bedside-to-bench patient-oriented translational clinical investigation. Arch Dermatol Res. 2009;301:65-70. doi: 10.1007/s00403008-0890-x. 
2. Vedove CD, Del Giglio M, Schena D, Girolomoni G. Drug-induced lupus erythematosus. Arch Dermatol Res. 2009;301:99105. doi: 10.1007/s00403-008-0895-5.

3. Michaelis TC, Sontheimer RD, Lowe GC. An update in drug-induced subacute cutaneous lupus erythematosus. Dermatol Online J. 2017;23:13030/qt55×42822.

4. He Y, Sawalha AH. Drug-induced lupus erythematosus: an update on drugs and mechanisms. Curr Opin Rheumatol. 2018;30:490-7. doi: 10.1097/BOR.0000000000000522.

5. Lowe GC, Henderson CL, Grau RH, Hansen CB, Sontheimer $\mathrm{RD}$. A systematic review of drug-induced subacute cutaneous lupus erythematosus. Br J Dermatol. 2011;164:465-72. doi: 10.1111/j.1365-2133.2010.10110.x.

6. Bolognia J, Schaffer J, Cerroni L, editors. Dermatology: volume I. 4th ed. Philadelphia: Elsevier; 2018. 